\title{
Increasing Trend of Clinical Laboratory Testing at Gelephu Central Regional Referral Hospital, Bhutan
}

\author{
Vishal Chhetri*, Karma Yangchen, Chenga Dawa \\ Clinical Laboratory Unit, Centre Regional Referral Hospital, Gelephu, Bhutan
}

*Corresponding author -

\author{
Vishal Chhetri \\ Clinical Laboratory Unit, Centre Regional Referral Hospital, Gelephu, Bhutan \\ Emailid-vishal_srmc@yahoo.com
}

\begin{abstract}
Bhutanese population have access to both traditional and modern medicine. The record published in Annual Health Bulletin (AHB), Ministry of Health $(\mathrm{MoH})$ recorded the number of people seeking modern medicine has drastically escalated over the past few years. Gelephu based Central Regional Referral Hospital (CRRH), established in 1960's, sees the second highest number of outpatient department OPD cases in the country. As a result the number of clinical laboratory testing has also increased over the time. The ratio of annual OPD attendance and annual laboratory test recorded at the medical record and AHB depicted at the ratio of 1:1 until 2013 and the ratio increased to 1:1.9 in 2017, significant at $p<0.05$. The annual total laboratory test increased from $n=118567$ in 2013 to $n=234869$ in 2017 , with almost an increase by average of $2 \%$ annually. The increasing number of test order, increases the probabilities for error. Hence, laboratory quality system is of paramount importance. The introduction of automation in Biochemistry (2014) and Hematology (2015), has significantly reduced analytical errors and has generated reliable, and quality assured reports. No reports have been published until now from CRRH to indicate the increasing clinical laboratory tests. Therefore, the data analysis from this study will present the ever increasing annual laboratory testing conducted at CRRH and would also provide the national medical procurement and supply division with information critical for annual supply forecasting.
\end{abstract}

\section{Introduction}

Modern medicine or Western medicine is a way of health professionals treating diseases using drugs, radiation, or surgery, also called allopathic medicine. ${ }^{[1]}$ The diagnostic services such as clinical laboratory and medical imaging science remains the heart of the system. Clinical laboratory services is a multi-disciplinary specialty comprising of clinical hematology, biochemistry, microbiology, molecular and sero-diagnostics, cytology, histopathology, blood banking, parasitology and allied health services. Clinical laboratory have direct impact on patients. Almost $70 \%$ of medical diagnosis are based from laboratory results. ${ }^{[2]}$ Laboratory information enables healthcare professionals to make timely and appropriate evidence-based diagnostic and therapeutic decisions for their patients. ${ }^{[3]}$

The Kingdom of Bhutan located between the two giant nations, India to the south and China in the north, with approximate population of 800,000 , enjoys free health services under its farsighted kingship. Bhutan's heath sector has made commendable successes towards achieving its State Policy, which states "State shall provide free access to basic health services". Hence, the life expectancy of Bhutanese population has increased from 32.4 in 1960 to 69.5 in $2015 .{ }^{[4]}$ The modern health in Bhutan started with first medical doctor in 1954 and currently has 31 hospitals, 235 Basic Health Units and 562 Outreach clinics to reach all the population. ${ }^{[5]}$ Located at the southern foothills, Gelephu CRRH caters to the need of patients from Sarpang, Tsirang, Dagana, Zhemgang and Pemagatshel Districts. The hospital laboratory has been recently equipped with auto machine for hematology, biochemistry, blood component preparation and qualified medical lab technicians/technologist. The Clinical laboratory services results are a vital component for all the patients attending hospital for either simple fever or for complicated illnesses, potentially life threatening situations; including end-of-life care. ${ }^{[3]}$

Though the AHB published by $\mathrm{MoH}$, Thimphu, reflected the annual OPD attendance but has not published the annual laboratory test conducted comprehensively. Therefore, this retrospective survey study aims to present the ever increasing annual laboratory test conducted at CRRH and provide the national laboratory procurement and supply division with information critical for annual supply forecasting.

\section{Methodology}

The clinical laboratory unit at Gelephu CRRH offers; Biochemistry, Blood bank, Microbiology, Cytology, Hematology, Serology and Parasitology services for all the OPD, emergency and ward-patients. Samples such as blood, urine, sputum, stool and body fluids are collected and processed in accordance to the Standard Operation Procedure (SOP). The annual laboratory test record is available from annual census maintained with the medical 
record unit of hospital and published in $\mathrm{AHB}, \mathrm{MoH}$ (http://www.health.gov.bt).

Data entry is done using Microsoft excel and analyzed using SPSS. Descriptive information is presented as frequencies, means and percentages. P-value of $\leq 0.05$ is considered statistically significant.

\section{Results and discussion}

The OPD at CRRH, Gelephu examines the second highest number of patients in the country and it keeps rising every year. ${ }^{[4,6]}$ The ratio of annual OPD attendance and annual laboratory test was $1: 1$ until 2013 but the ratio increased to $1: 1.9$ in 2017 , statistically significantly at $p<0.05$.

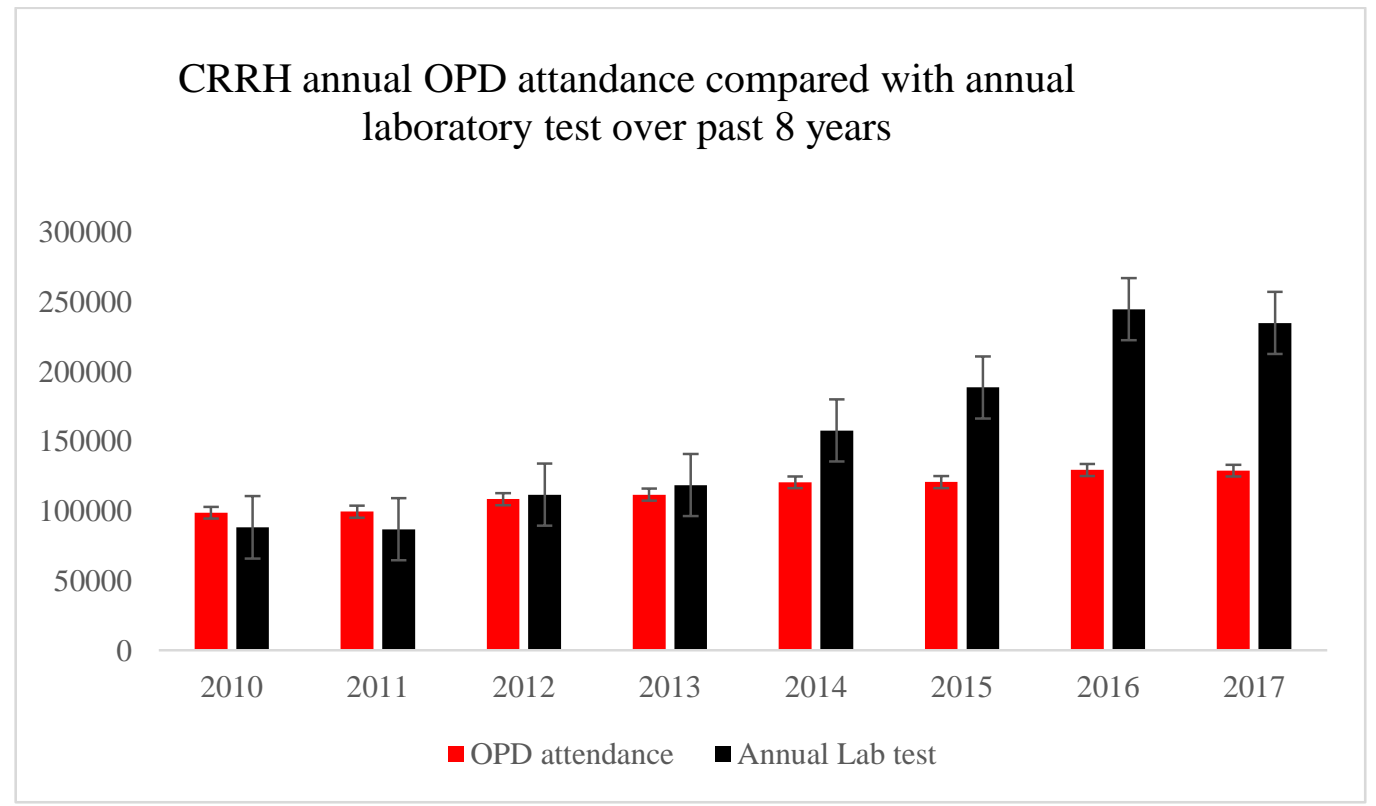

Figure 1: Annual OPD attendance v/s annual clinical laboratory testing conducted at Gelephu CRRH over past 8 years.

The clinical laboratory unit at CRRH felt the increasing pressure of patient load visiting the hospital and seeking laboratory service. The annual total laboratory test increased from $n=118567$ in 2013 to $n=234869$ in 2017 , with average increasing rate of $2 \%$ annually. The increasing number of specialist at hospital could be another factor related to increasing laboratory tests. Therefore, the quality of laboratory test result is of paramount important to meet this ascending demand.

The biochemistry unit recorded the highest number of test conducted and it increased drastically from 2014 onwards ( $n=76829$ ) as compared to just $n=40960$ in 2013 . The basic blood biochemistry tests, such as; blood glucose, liver function test (LFT), renal function test (RFT), lipid profile, total protein, albumin, uric acid, gamma-glutamyl transferase (GGT), electrolytes and special tests such as creatine kinase (CK), CK$\mathrm{MB}, \mathrm{HbA1C}$, iron, lactate dehydrogenase (LDH), magnesium, phosphorus were started since the introduction of auto analyzer in 2014. The use of automation for biochemistry has drastically assisted the analytical process, increasing the efficacy and reducing turn-around-time (TAT) ${ }^{[5]}$ The available auto machines (EM360 and EM 200), under rental reagent scheme (RRS) is based on the principle of photometry and light transmittance at various wavelength. The blood sugar (fasting/post-prandial and random samples) is the highest test that is been conducted because of increasing trend of non-communicable diseases in Bhutan, including Diabetes Mellitus (DM). ${ }^{[8]}$

The use of photometer prior to introduction of auto machine had poor performance both in the internal quality control (IQC) and external quality assurance scheme (EQAS). The introduction of auto analyzer improved IQC and EQA performances. The biochemistry unit follows the quality control (QC) rules as stated by James Westgard, using Levy Jennings (LJ) chart ${ }^{[9]}$ with two level control materials. The biochemistry unit participated in EQA conducted by Royal College of Pathology Australian (RCPA) from 2013 to 2016 and currently participates in Pacific Paramedic Training Centre (PPTC) scheme. All the serological and immunology test are recorded under biochemistry and performed with rapid test kits (RDT). 


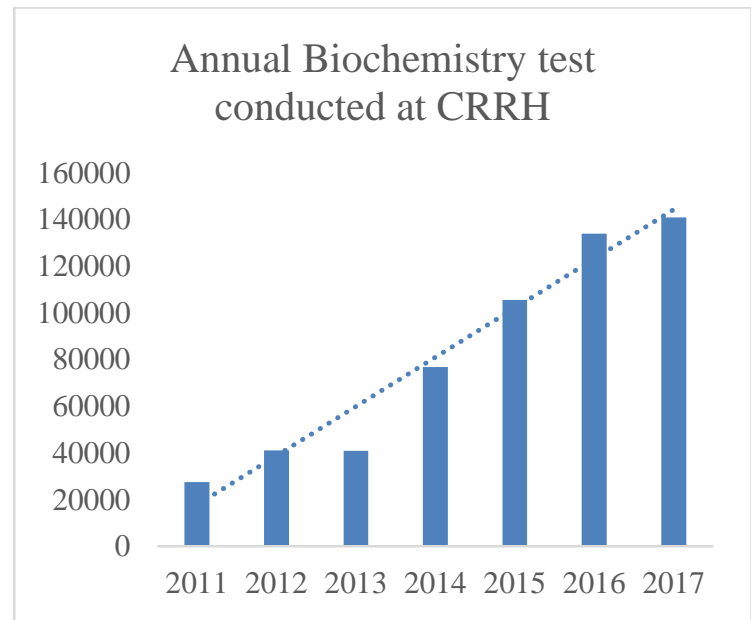

Figure 2: Annual increasing biochemistry blood test conducted at CRRH. There is significant increase since the introduction of automation in 2014.

\section{Annual average IQC CV in Biochemistry}

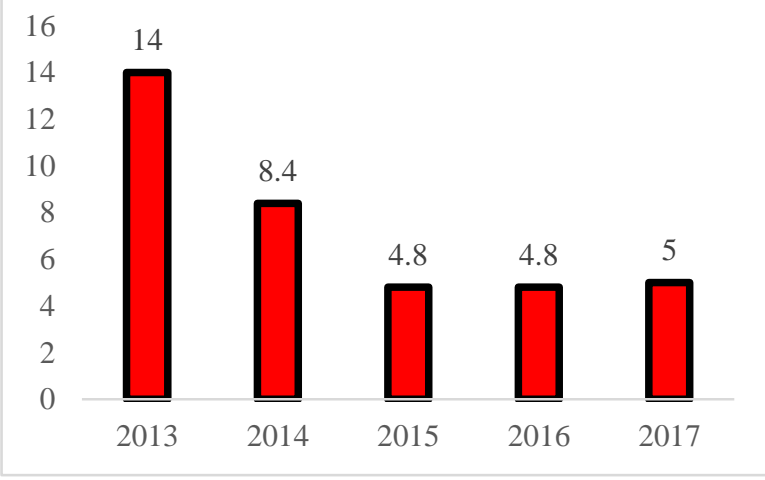

Figure 3: Annual average IQC CV of biochemistry unit with Precinorm and Precipath. In 2013 annual average CV was 14 (photometer meter was used for measurement), it was brought down to 8.4 in 2014 after introduction of automation and then IQC consistently improved.

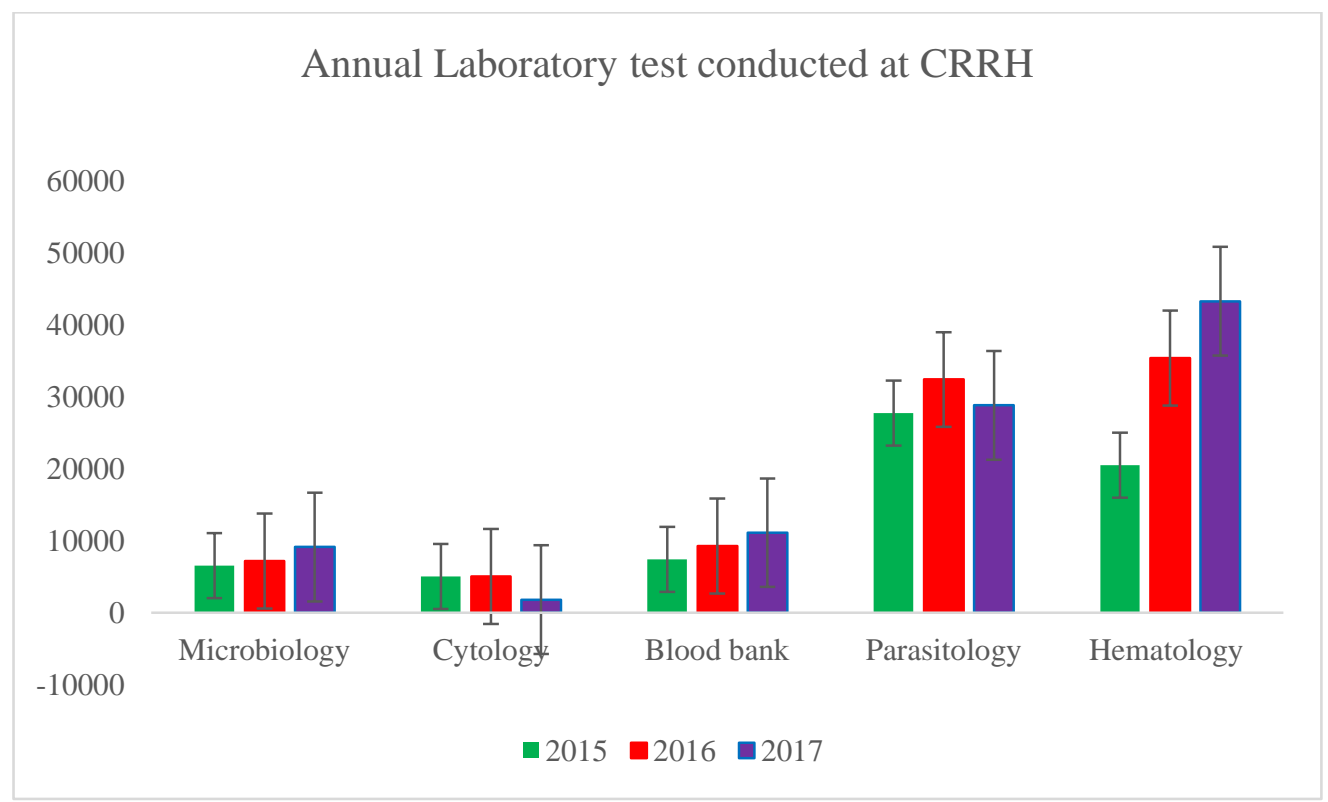

Figure 4: Annual laboratory test done in CRRH from 2015 to 2017. Almost all the units; Microbiology, Hematology and Blood Bank recorded annual increase in the test. The Cytology unit recorded relatively low number of cases in 2017, this is due to non-availability of pathologist in the center and establishment of additional cytology center in the region.

The microbiology laboratory tests all the samples (urine, pus, stool, blood, body fluids, and sputum) in accordance to the clinical and laboratory standard institute (CLSI) guideline. ${ }^{[10]}$ Antibiotic susceptibility testing (ABST) is performed for the isolates and the result break points are interpreted according to CLSI guidelines. ${ }^{[11]}$ The primary isolates are inoculated into Mueller-Hinton agar plates, then disc diffusion method according to Kirby-Bauer to study ABST. For the Neisseria species chocolate agar plates, according to the calibrated dichotomous sensitivity (CDS) method is performed. ${ }^{[12]}$ The microbiology unit tested about $n=6534$, $\mathrm{n}=7156, \mathrm{n}=9105$ in 2015,2016 and 2017 respectively, with the increasing number of test every year. IQC is done using ATCC 25922 (E. coli), ATCC 27853 (Pseudomonas aeruginosa) for Gram negatives and ATCC 25923 (Staphylococcus aureus) for Gram positive isolates. The microbiology unit participates in PPTC microbiology EQA program since 2016.
The microbiology unit also screens for tuberculosis caused by acid fast bacilli (AFB) Mycobacterium tuberculosis with Ziehl-Neelsen (ZN) smear microscopy technique. ${ }^{[13,14]}$ Recently more rapid and sensitive nucleic acid amplification (NAA) GeneXpert technique has been introduced in 2016 for high sensitivity detection and to study rifampicin resistance related mutations. ${ }^{[13,15]}$ The TB laboratory participates in EQA programme conducted by Royal Centre for Disease control (RCDC); such as blinded rechecking and competency panel testing. ${ }^{[16,18]}$

The Hematology analysis at CRRH is done using Sysmex XS$1000 \mathrm{i}$, a fully automated analyzer under the RRS scheme introduced in 2015. Earlier hematology tests, such as total WBC count, platelet count and WBC differential count were performed manually. But with the introduction of fully automated analyzer, complete blood count (CBC) study with 21 test parameters is 


\section{International Journal of Innovative Research in Medical Science (IJIRMS) Volume 03 Issue 11 Nov 2018, ISSN: 2455-8737, Imp. Factor - 4.102 Available online at $-\underline{w w w . i j i r m s . i n}$}

performed. The other tests performed at hematology unit are erythrocyte sedimentation rate (ESR), reticulocytes count, absolute eosinophil count (AEC), coagulation test, body fluid analysis and special test such as peripheral blood smear (PBS) interpretation performed by trained medical technologist. The total hematology testing increased to $n=35331$ in 2016 as compared to $n=20446$ in 2015. The automation was installed towards the end of 2015. The hematology unit performs in-house IQC with Delta check method and does not participate in any EQA programme. ${ }^{[18]}$

Routine urine examination, urine acetone, pregnancy test, stool occult blood test and stool microscopy for parasite is conducted at parasitology unit following SOP ${ }^{[19]}$ During emergencies rapid test kits are used to perform urine analysis. The cytology unit screens all Pap smear for early detection of cervical cancer for the slides collected from the CRRH and catchment Basic Health units (BHU). It is stated that community-based cervical cancer screening by testing self-collected samples for human papillomavirus (HPV) can achieve high coverage in rural Bhutan. ${ }^{[20]}$ The total cytology test decreased in 2017 as compared to 2015, due to the unavailability of pathologist and opening of cytology center at neighboring district.

Routine blood bank (BB) activity includes collection of blood from voluntary and replacement blood donors, cross matching of blood for transfusion and blood grouping. ${ }^{[21]}$ Since 2013 the BB started providing blood component services. All the blood bank procedure are carried as per the national standards. ${ }^{[22]}$ There has been increasing number of blood donors, inclusively voluntary blood donors recorded at CRRH. ${ }^{[17]}$ The blood bank conducts all the daily IQC (cell grouping, serum grouping, control cells) and also participates in the EQA in blood grouping serology (BGS) conducted by JDWNRH ${ }^{[21]}$ and also in PPTC scheme.

Though working in laboratory is not totally safe but the use of personal protective equipment (PPE) and good laboratory practice (GLP) could reduce the associated risk. Laboratory analysis is a complex process involving both high end technology and requires daily update of knowledge. Clinical laboratories have long focused their attention on quality control methods and quality assessment programs dealing with analytical aspects of testing.

\section{Laboratory QA System}

The nature of clinical laboratory services is changing under the combined pressures of increasing workloads, cost constraints and technological advancement. So it is necessary to create and maintain robust quality assurance to ensure appropriate use of the systems. QA is an essential part of medical laboratory process for systematic monitoring of operation in a clinical laboratory. It consists of IQC and EQAS. ${ }^{[24,25]}$ The IQC includes SOP, preanalytical, analytical and post-analytical features. ${ }^{[26]}$ EQAS involves evaluation of a number of laboratories by an outside agency on the performance of a number of laboratories based on their analytical performance of tests on samples supplied by the external agency. ${ }^{[27]}$ IQC identifies precision and EQAS identifies accuracy of clinical laboratory. CRRH participated in RCPA (biochemistry) EQA programme, though the annual average score was initially low, but the score improved gradually (data not shown due to incomplete record) when compared among peer group.
The laboratory errors are classified as; pre-analytical, analytical or post-analytical error and the highest incidence of laboratory errors occurs in the pre-analytical phase. ${ }^{[28,29]}$ Though several preanalytical errors event occurred in our laboratory, there is no recorded data of the total error occurred. The post analytical error are mostly reduced with the involvement of IT and the CRRH laboratory has interfaced Poytech laboratory information system (LIS), though the effective introduction of IT system remains challenging. ${ }^{[30]}$ The analytical errors are minimized with strict IQC and EQA system, and also with the use of sophisticated automation with regular calibration.

\section{Conclusion}

The study shows that there is annual increase in both the OPD attendance and number of laboratory test performed, significantly at $p<0.05$. All the laboratory unit; Microbiology, Biochemistry, Hematology and Blood bank recorded the increasing number of annual laboratory test at average increasing rate of $2 \%$ annually except in cytology that recorded decreasing annual test due to unavailability of pathologist and opening of cytology center at neighboring districts.

To meet the demand of increasing laboratory tests, the introduction of automation and IT has drastically assisted the analytical and post-analytical process, increasing the efficacy and reducing TAT. To ensure the reliability of laboratory results and reduce the risk of errors, $98 \%$ of the CRRH clinical laboratory staff are trained in basic QA/QC and refresher trainings conducted annually.

Considering the large number of patients with infectious and communicable diseases visiting clinical laboratory the application of infection control measures to prevent nosocomial infection should be initiated. The limitations of this study are such as, no proper record of laboratory error incidence, no proper record of test conducted before 2014 and inconsistent supply of QC material. Finally, this study would provide the national laboratory procurement and supply division with information critical for annual supply forecasting.

\section{Reference}

[1] Singh, A., Modern medicine: Towards prevention, cure, well-being and longevity. Revista Latinoamericana de Psicopatologia Fundamental, 2010. 13(2): p. 265-282.

[2] Fowler, D., T. Martin, and L. Spence, The clinical laboratory practitioner. Clinical Laboratory Science, 2005. 18(4): p. 199.

[3] Forsman, R., The value of the laboratory professional in the continuum of care. Clinical leadership \& management review: the journal of CLMA, 2002. 16(6): p. 370-373.

[4] Bhutan Annual Health Bulletin 2018 http://www.health.gov.bt/wpcontent/uploads/ftps/annual-healthbulletins/Annual\%20Health\%20Bulletin-2018. Accessed on 25th September 2018.

[5] Tobgay, T., et al., Progress and delivery of health care in Bhutan, the land of the thunder dragon and gross 


\section{International Journal of Innovative Research in Medical Science (IJIRMS) Volume 03 Issue 11 Nov 2018, ISSN: 2455-8737, Imp. Factor - 4.102 Available online at - $\underline{w w w . i j i r m s . i n}$}

national happiness. Tropical Medicine \& International Health, 2011. 16(6): p. 731-736.

[6] Bhutan Annual Health Bulletin 2017. www.health.gov.bt/wp.../annual-health-

bulletins/Annual\%20Health\%20Bulletin-2017. Accessed on 25th September 2018.

[7] Hawker, C.D., Laboratory automation: total and subtotal. Clinics in laboratory medicine, 2007. 27(4): p. 749-770.

[8] Zam, K., et al., A first country-wide review of Diabetes Mellitus care in Bhutan: time to do better. BMC health services research, 2015. 15(1): p. 389.

[9] Westgard, J.O., et al., A multi-rule Shewhart chart for quality control in clinical chemistry. Clin Chem, 1981. 27(3): p. 493-501.

[10] Wayne, P., Clinical and laboratory standards institute. Performance standards for antimicrobial susceptibility testing. 2011.

[11] Adeep, M., et al., A retrospective analysis of the etiologic agents and antibiotic susceptibility pattern of uropathogens isolated in the Jigme Dorji Wangchuck National Referral Hospital, Thimphu, Bhutan. BMC research notes, 2016. 9(1): p. 54.

[12] Tshokey, T., et al., Burden of Gonorrhea and Trends in Antibiotic Susceptibility Pattern of Neisseria gonorrhoea in Bhutan Over Four Years (2012-15).

[13] Agrawal, M., et al., Comparative study of GeneXpert with ZN stain and culture in samples of suspected pulmonary tuberculosis. Journal of clinical and diagnostic research: JCDR, 2016. 10(5): p. DC09.

[14] Wangdi, K. and M.R. Gurung, The epidemiology of tuberculosis in Phuentsholing General Hospital: a sixyear retrospective study. BMC research notes, 2012. 5(1): p. 311.

[15] Ioannidis, P., et al., Cepheid GeneXpert MTB/RIF Assay for Mycobacterium tuberculosis detection and rifampin resistance identification in patients with high clinical suspicion of TB and smear negative microscopy. Journal of clinical microbiology, 2011: p. JCM. 00718-11.

[16] Organization, W.H., National tuberculosis control programme, Bhutan. 2007, WHO Regional Office for South-East Asia.

[17] Chhetri, V., Sero-prevalence of Transfusion Transmitted Infections among Blood Donors at CRRH, Gelephu, Bhutan. Vol. 6. 2018.
[18] Jamtsho, R., Stability of lyophilized human serum for use as quality control material in Bhutan. Indian Journal of Clinical Biochemistry, 2013. 28(4): p. 418-421.

[19] Norbu, K., et al., Prevalence of intestinal parasitic infection in patients attending National Referral Hospital, Thimphu, from 2013 to 2015: A retrospective study. Bhutan Health Journal, 2018. 4(1): p. 46-49.

[20] Baussano, I., et al., Cervical cancer screening in rural Bhutan with the careHPV test on self-collected samples: an ongoing cross-sectional, population-based study (REACH-Bhutan). BMJ open, 2017. 7(7): p. e016309.

[21] Chhetri, V., Frequency Distribution of $A B O$ and $R h$ blood group among Blood Donors at Centre Regional Referral Hospital, Gelephu: A Retrospective Study. Vol. 6. 2018.

[22] Chejor, P., Blood and Blood Products Regulation in Bhutan: Progress and Challenges. J Med Sci and Clin Res, 2018. 6(05): p. 1128-32.

[23] Soisangwan, P., External quality assessment in blood group serology in the World Health Organization SouthEast Asia Region. WHO South-East Asia journal of public health, 2012. 1(4): p. 423.

[24] Jamtsho, R., et al. The process for implementation of External Quality Assessment Scheme in Clinical Chemistry for District Laboratories in Bhutan. in International Conference on The Changing Environment Challanges for Society. 2010.

[25] McPherson, R.A. and M.R. Pincus, Henry's Clinical Diagnosis and Management by Laboratory Methods EBook. 2017: Elsevier Health Sciences.

[26] Endröczi, E., Quality assurance in clinical laboratory diagnosis. Orvosi hetilap, 1990. 131(45): p. 2463-2467.

[27] Jamtsho, R. and W. Nuchpramool, Implementation of External Quality Assessment Scheme in clinical chemistry for district laboratories in Bhutan. Indian journal of clinical biochemistry, 2012. 27(3): p. 300-305.

[28] Laposata, M. and A. Dighe, "Pre-pre" and "post-post" analytical error: high-incidence patient safety hazards involving the clinical laboratory. Clinical Chemical Laboratory Medicine, 2007. 45(6): p. 712-719.

[29] Plebani, M., Errors in clinical laboratories or errors in laboratory medicine? Clinical Chemistry and Laboratory Medicine (CCLM), 2006. 44(6): p. 750-759.

[30] Jones, R.G., O.A. Johnson, and G. Batstone, Informatics and the clinical laboratory. The Clinical Biochemist Reviews, 2014. 35(3): p. 177. 